\title{
Treatment patterns in diabetic macular edema in Taiwan: a retrospective chart review
}

This article was published in the following Dove Press journal: Clinical Ophthalmology

\author{
Shwu-Jiuan Sheu ${ }^{1,2}$ \\ Cheng-Kuo Cheng ${ }^{3,4}$ \\ Hsi-Kung Kuo ${ }^{5}$ \\ Ching-Yao Tsai ${ }^{2,6}$ \\ Tai-Chi Lin ${ }^{2,7}$ \\ Jonathan $\operatorname{Tan}^{8}$ \\ Hitesh Chandwani ${ }^{8}$ \\ Michael Adena ${ }^{9}$ \\ Shih-Jen Chen ${ }^{2,7}$
}

'Department of Ophthalmology, Kaohsiung Veterans General Hospital, Kaohsiung, Taiwan; ${ }^{2}$ Department of Ophthalmology, School of Medicine, National Yang-Ming University, Taipei, Taiwan; ${ }^{3}$ Department of Ophthalmology, Shin-Kong Wu Ho-Su Memorial Hospital, Taipei, Taiwan;

${ }^{4}$ Department of Medicine, School of Medicine, Fu-Jen Catholic University, New Taipei, Taiwan; ${ }^{5}$ Department of Ophthalmology, Kaohsiung Chang Gung Memorial Hospital, Kaohsiung, Taiwan; ${ }^{6}$ Department of Ophthalmology, Taipei City Hospital Zhongxing Branch, Taipei, Taiwan; ${ }^{7}$ Department of Ophthalmology, Taipei Veterans General Hospital, Taipei, Taiwan; ${ }^{8} \mathrm{Global}$ Health Economics and Outcomes Research, Allergan Singapore Pte. Ltd., Singapore, Singapore; ${ }^{9}$ Datalytics Pty Ltd, Kingston, ACT, Australia
Correspondence: Shih-Jen Chen Taipei Veterans General Hospital, 20I, Shihpai Road, Beitou District, Taipei, Taiwan

Fax +88622875 7/ 33

Email sjchen96@gmail.com
Objectives: To characterize diabetic macular edema (DME) treatment patterns in Taiwan and examine their impact on health care resource utilization and visual and anatomic outcomes.

Methods: Retrospective, observational cohort study of longitudinal data from medical records of five hospital ophthalmology clinics. Patients with type 2 diabetes and DME who received $\geq 1$ laser treatment or pharmacotherapy (intravitreal/subtenon corticosteroids and/or intravitreal anti-vascular endothelial growth factor [VEGF] agents) between January 2012 and December 2013 (index period) and attended $\geq 1$ follow-up visit after the first treatment during that period were identified (prevalent population, $\mathrm{N}=431$ ). In addition, a subset that received no anti-VEGFs before 2012 (anti-VEGF-naïve population, N=77) was analyzed. Outcome measures were change in DME treatment distribution between January 2009 and December 2014 and health care resource utilization over up to 3 years from the first DME treatment received in the index period (prevalent population), mean number of anti-VEGF injections and change from baseline in visual acuity and central macular thickness over 12 months (anti-VEGF-naïve population). Results: Between 2009 and 2014, laser treatment use declined, overall use of anti-VEGFs increased, and bevacizumab use decreased proportionately as ranibizumab use increased. Patients receiving corticosteroids and anti-VEGFs in the first 6 months post-index had greater health care resource utilization than those treated with laser, corticosteroids, or anti-VEGF alone $(P<0.0001$, cross-cohort comparison). Among anti-VEGF-naïve patients, $69 \%$ received one to four antiVEGF injections in the first year post-index. Overall, visual acuity improvement from baseline was minimal at 1 year ( 0.4 letters, observed data; 0.1 letters, last observation carried forward), and modest central macular thickness reduction ( $28 \mu \mathrm{m}$ [last observation carried forward]) was detected.

Conclusion: In Taiwanese clinics, DME treatment patterns have shifted from use of laser to anti-VEGFs (with higher health care resource utilization); however, few patients receive antiVEGF injections at the frequency reported in landmark trials, consistent with poorer visual outcomes. Effective alternative treatments with lower treatment burden should be considered. Keywords: macular edema, diabetes, treatment patterns, treatment outcome, anti-vascular endothelial growth factor, corticosteroid

\section{Introduction}

The global prevalence of diabetes mellitus among people aged 20-79 years is expected to increase by $20 \%$ in 2030 , compared with $2010 .{ }^{1}$ This rapid growth poses a worldwide health and economic challenge, ${ }^{2}$ in part because common microvascular complications of diabetes mellitus (diabetic retinopathy and diabetic macular edema [DME]), ${ }^{2,3}$ are leading causes of visual impairment in developed countries. ${ }^{2-5}$ Consistent with this assessment, an analysis of the Taiwan Longitudinal Health Insurance Database revealed that, among patients with type 2 diabetes, the number 
of patients with sight-threatening diabetic retinopathy and DME increased between 2005 and 2011 (women, from 576 to 1,142 [98\%]; men, from 668 to 1,149 [72\%]), correlating with a mean annual increase of $10.1 \%$ in the diabetic population. ${ }^{6}$ Similar figures were found in a study conducted in Europe. ${ }^{7}$

The expansion of the diabetic population has resulted in an increased demand for resources to treat diabetic retinopathy and DME. ${ }^{8}$ The direct medical cost of a patient with DME was shown to be 3.1 times higher than the national average medical expenditure per person in Taiwan between 2004 and 2009. ${ }^{9}$ In the United States, direct medical costs from 2000 to $2004^{10}$ and 1999 to $2004^{11}$ were reported to be $30 \%$ and $75 \%$ higher in diabetic patients with DME, compared with those without diabetic retinopathy ${ }^{10}$ or DME, ${ }^{11}$ respectively.

DME has traditionally been treated with laser photocoagulation, but treatment patterns have evolved since the introduction of intravitreal treatments, which include anti-vascular endothelial growth factor (VEGF) agents and corticosteroids. ${ }^{4}$ Landmark Phase III/IV clinical trials, in which patients received an average of seven to ten intravitreal injections per eye in the first year, have demonstrated that anti-VEGF agents, such as bevacizumab (Avastin ${ }^{\circledR}$, Genentech Inc., San Francisco, CA, USA), ranibizumab (Lucentis ${ }^{\circledR}$, Genentech Inc.), and aflibercept (Eylea ${ }^{\circledR}$, Regeneron Pharmaceuticals Inc., Tarrytown, NY, USA), are effective in improving visual and anatomic outcomes in patients with DME, including those of Asian descent. ${ }^{12-15}$ Based on these results, anti-VEGF agents have been approved as DME treatment in many countries. In Taiwan, ranibizumab and aflibercept have been reimbursed nationally since 2013 and 2016, respectively, although patients must meet specific criteria (optical coherence tomography [OCT]-defined central retinal thickness $[\geq 300 \mu \mathrm{m}]$ and visual acuity [VA, 20/40-20/400]) to be eligible. Intravitreal corticosteroid treatment for DME largely consisted of offlabel triamcinolone (eg, Kenacort ${ }^{\mathrm{TM}}$, Bristol-Myers Squibb, Taipei, Taiwan) until 2016. ${ }^{16}$ However, a sustained-release dexamethasone intravitreal implant (Ozurdex ${ }^{\circledR}$, Allergan plc, Dublin, Ireland) with efficacy up to 6 months ${ }^{17}$ was approved for use in March 2016.

Although several intravitreal agents are used for the treatment of DME in Taiwan, data on DME treatment patterns are limited. Accordingly, the objective of this study was to characterize the patterns of DME treatment in Taiwan and to examine their impact on health care resource utilization and visual and anatomic outcomes.

\section{Methods \\ Study design, data source, and patient identification}

This was a retrospective, observational cohort study of longitudinal data from medical records of patients with DME who were treated at five hospital ophthalmology clinics (one public and one private in Southern Taiwan; two public and one private in Northern Taiwan) between January 1, 2012 and December 31, 2013. Inclusion of up to 120 patients from each of the five participating hospitals was planned for analysis. Data were captured in an electronic database and deidentified. Data extraction was conducted between October and December 2014. Approvals from the institutional review boards of the following hospitals were obtained prior to study initiation: Kaohsiung Veterans General Hospital (Shwu-Jiuan Sheu), Shin-Kong Wu Ho-Su Memorial Hospital (ChengKuo Cheng), Chang Gung Medical Foundation (Hsi-Kung Kuo), Taipei City Hospital (Ching-Yao Tsai), and Taipei Veterans General Hospital (Tai-Chi Lin; Shih-Jen Chen). The need for written patient consent was waived in light of the retrospective study design, nature of the data collected, and low risk of confidentiality breach.

Available medical records from January 1, 2009, to December 31, 2014, were screened to identify patients (prevalent population) who had 1) a diagnosis of DME; 2) received $\geq 1$ laser treatment or pharmacotherapy (intravitreal or subtenon corticosteroids and/or intravitreal antiVEGF agents) between January 2012 and December 2013 (index period); and 3) attended $\geq 1$ follow-up clinic visit after the first treatment administered during that period. Patients with concurrent progressive retinal disease (eg, neovascular age-related macular degeneration [AMD] and retinal vein occlusion [RVO]) requiring treatment to prevent vision loss, or who were already participating in a clinical trial, were excluded. Patients with concurrent stable retinal disease (eg, RVO or dry AMD) not requiring treatment to prevent vision loss were included at the investigator's discretion.

Further screening identified a subset of patients (antiVEGF-naïve population) with 1) a diagnosis of DME and first anti-VEGF injection in the 2012-2013 index period; 2) no anti-VEGF therapy before 2012 ; 3 ) known corrected VA at baseline $\leq 20 / 40$ and $\geq 20 / 320$ Snellen (ie, 40-85 Early Treatment Diabetic Retinopathy Study [ETDRS] letters) between Day -30 and Day 7 of the index date (date of the first anti-VEGF injection in the index period); 4) VA measured $\geq 60$ days post-index; 5) known follow-up for at least 12 months from baseline; and 6) no RVO or AMD diagnosis. 


\section{Study outcomes}

Sociodemographic factors and clinical measures recorded at the time of the first treatment between January 1, 2009, and December 31, 2013, including VA, treatment history, and lens status, were collected as baseline data for each patient. If a characteristic was not recorded in that period, a search of data from the 12 months following (preferentially) or preceding the baseline visit was conducted. Characteristics not documented during the 12-month extension period were categorized as "missing". Data collected at each clinic visit included VA of each eye, central macular thickness (CMT) measured by OCT, treatment administered, and whether fluorescein angiography was performed. VA was originally recorded in Snellen and converted to the ETDRS letter scores using a published algorithm. ${ }^{18}$ To aid in the comparison of CMT assessed with different types of OCT imaging techniques, measurements were transformed to Stratusequivalent values using published algorithms..$^{19,20}$

In the anti-VEGF-naïve population, baseline VA and CMT were measured between Day 30 pre-index and the date closest to the index date; if no measurements were available before the index date, the closest measurements $\leq 7$ days after the index date were used. The VA and CMT at the date closest to 365 days (between days 330 and 419) post-index treatment were the 1-year follow-up values; if those values were not available, the most recent VA and CMT values obtained between days 8 and 329 after the index date were used (as last observation carried forward [LOCF]).

Outcomes assessed in the prevalent population included the change in DME treatment distribution between January 2009 and December 2014, as well as health care resource utilization over up to 3 years from the date of the first DME treatment received in the index period. Health care resource utilization, evaluated in terms of frequency of clinic visits, DME treatment visits, and assessment visits during which OCT and fluorescein angiography were performed, was analyzed according to the type of DME treatment received in the first 6 months post-index (ie, no intravitreal injection/ laser alone, or injection of corticosteroid [ \pm laser], anti-VEGF [ \pm laser], or both [ \pm laser]). Outcomes assessed in the antiVEGF-naïve population were the mean number of anti-VEGF injections administered in the first year post-index overall and per quarter year, as well as the change from baseline in VA and CMT during that year.

\section{Data analyses}

Descriptive statistics were used to summarize patient demographic and disease-related data. Chi-square and $t$-tests were conducted to analyze categorical and continuous variables, respectively. Analyses of VA and CMT were undertaken using observed values and repeated with the LOCF method for imputation of missing values (provided the last observation was obtained $\geq 8$ days after the index date). Multivariate regression analyses were used to explore the relationship between intravitreal treatment frequency and outcomes, as well as the mean resource utilization per quarter across treatment category, with adjustment for potential confounders (age, gender, baseline VA, DME bilateralism, and number of laser treatments) where appropriate. A formal sample size calculation was not conducted. All analyses were carried out using STATA software version 13 (StataCorp LP, College Station, TX, USA).

\section{Results}

Of 441 patients (783 study eyes) with DME who met the eligibility criteria, 431 patients (765 eyes) with type 2 diabetes were included in the analyses of the prevalent population. Ten patients ( 18 eyes) had type 1 diabetes and were excluded from analysis. In comparison, 77 patients (101 eyes) comprised the anti-VEGF-naïve population. Baseline demographics and clinical characteristics are shown in Table $1 .{ }^{18}$

\section{Treatment patterns and health care utilization in the prevalent population}

The absolute number of DME treatments administered over the study period and the change in distribution of individual DME treatments (as a percentage of total DME treatments) are presented in Figure 1A and B, respectively. The low number of treatments in the earlier years (2009-2011) is likely a result of the study design, as patients were required to have received anti-DME treatment during the 2012-2013 period, but not necessarily before 2012. Between 2009 and 2014, there was an overall decline and increase in the use of laser and anti-VEGF agents, respectively, whereas the use of corticosteroids remained relatively constant. Most notably, the use of bevacizumab decreased in proportion with the increase in ranibizumab use. Only six cases of aflibercept use were recorded, which reflects the fact that aflibercept became commercially available only in the first half of 2014.

Across all treatment categories, there were significantly $(P<0.001)$ more clinic visits, treatment visits, and OCT assessments per quarter in the first 6 months post-index, compared with months 7-12, Year 2, or Year 3 (Figure 2). Overall, patients receiving both anti-VEGF agents and corticosteroids during the first 6 months post-index attended the 
Table I Baseline demographics and disease characteristics

\begin{tabular}{|c|c|c|}
\hline Variable & $\begin{array}{l}\text { Prevalent } \\
\text { population, } \\
\mathrm{N}=43 \text { I patients } \\
\text { (765 eyes) }\end{array}$ & $\begin{array}{l}\text { Anti-VEGF-naïve } \\
\text { population, } \mathrm{N}=77 \\
\text { patients }^{\mathrm{a}} \\
(\mathrm{I} 01 \text { eyes) }\end{array}$ \\
\hline Gender, female, n (\%) & $201(47)$ & $41(53)$ \\
\hline Mean age (SD), years & $61.8(9.1)$ & $62.2(9.3)$ \\
\hline Range & $28-90$ & $34-86$ \\
\hline \multicolumn{3}{|l|}{ HbAlc, n (\%) } \\
\hline$<7$ & $72(17)$ & $13(17)$ \\
\hline $7-8$ & $106(25)$ & $28(36)$ \\
\hline $8-10$ & $110(26)$ & $21(27)$ \\
\hline$>10$ & $56(13)$ & $8(10)$ \\
\hline Data not available & $87(20)$ & $7(9)$ \\
\hline \multicolumn{3}{|l|}{$\begin{array}{l}\text { Duration of diabetes mellitus, } \\
\text { years, } \mathrm{n}(\%)\end{array}$} \\
\hline $1-5$ & $58(13)$ & $7(9)$ \\
\hline $6-10$ & $83(19)$ & $16(21)$ \\
\hline$>10$ & I76 (4I) & $24(3 \mathrm{I})$ \\
\hline Data not available & $114(26)$ & $30(39)$ \\
\hline \multicolumn{3}{|l|}{ DME characteristics } \\
\hline Median duration, months & 32 & 6.2 \\
\hline Center-involving, n (\%) & $643(84)$ & $96(96)$ \\
\hline Bilateral, n (\%) & $334(77)$ & $24(3 I)$ \\
\hline $\begin{array}{l}\text { Lens status, pseudophakic, } \\
\mathrm{n}(\%)^{\mathrm{b}}\end{array}$ & $173(23)$ & $17(17)$ \\
\hline History of glaucoma, n (\%) & $47(6)$ & $6(6)$ \\
\hline Median visual acuity, ETDRS & 50 & 50 \\
\hline \multicolumn{3}{|l|}{ letters ${ }^{\mathrm{b}, \mathrm{c}}$} \\
\hline Range & $0-89$ & $20-70$ \\
\hline Mean baseline CMT (SD), $\mu \mathrm{m}^{\mathrm{b}}$ & 338 (139) & $369(137)$ \\
\hline
\end{tabular}

Notes: ${ }^{a}$ Only patients with type 2 diabetes were included in the analysis. ${ }^{b} E y e-l e v e l$ data. 'Originally recorded in Snellen and converted to ETDRS letters. ${ }^{18}$

Abbreviations: CMT, central macular thickness; DME, diabetic macular edema; ETDRS, Early Treatment Diabetic Retinopathy Study; HbAlc, hemoglobin AIc; SD, standard deviation; VEGF, vascular endothelial growth factor.

greatest number of clinic and treatment visits and had the greatest number of OCT assessments per quarter, followed by patients receiving anti-VEGF agents alone. In contrast, patients receiving laser photocoagulation alone during the first 6 months post-index had the lowest number of visits and assessments per quarter overall (Figure 2).

\section{Injection frequency and clinical outcomes in the anti-VEGF-naïve population}

The anti-VEGF treatment frequency and visual and anatomic outcomes were assessed at the eye level in the anti-VEGFnaïve population. As shown in Figure 3A, 69\% of eyes received one to four anti-VEGF injections, $25 \%$ received five or six injections, and only $6 \%$ received seven or more injections (range: 7-9) during the first year post-index. Notably, the mean number (standard deviation [SD]) of anti-VEGF injections administered over this period was 3.72 (1.80). Furthermore, the injection frequency decreased markedly after the first quarter (Figure 3B). Although all eyes received at least one injection during the first quarter (including $77 \%$ that received three or four), $59 \%, 69 \%$, and $75 \%$ of eyes received no injection during the second, third, and fourth quarters, respectively.

The mean observed VA (SD) was 51.2 (16.1) letters at baseline ( $\mathrm{n}=83$ eyes) vs 51.6 (21.9) letters at 1 year post-index ( $\mathrm{n}=83$ eyes), yielding a mean VA change from baseline of 0.4 (18.8) letters (Figure 4). ${ }^{12-14,18}$ However, the VA change from baseline improved at 3, 6, and 9 (peak) months, before declining at 1 year post-index (Figure 4). Results were similar at 6,9 , and 12 months after imputation of missing values $(n=101$ eyes; Figure 4$)$. The findings did not point to an association between the number of anti-VEGF injections received in the first year post-index and VA improvement. In addition, whether patients had at least one visit every 3 months during that period did not correlate with VA improvement; the mean VA change from baseline (SD) was -0.4 (18.1) letters for those who did attend $\geq 1$ visit every 3 months vs 1.8 (17.9) letters for those who did not.

Overall, the mean CMT change from baseline (SD) improved at $3(-59[137] \mu \mathrm{m})$ and remained constant through months $6(-56[142] \mu \mathrm{m})$ and $9(-56[131] \mu \mathrm{m})$. At 1 year post-index, the mean CMT change from baseline was smaller $(-28[117] \mu \mathrm{m})$, which was consistent with the observed decline in mean VA change from baseline discussed earlier. Findings pointed to an association between the number of anti-VEGF injections received in the first year post-index and the magnitude of the change in CMT from baseline (Figure 5). In addition, patients who attended $\geq 1$ visit every 3 months during that period exhibited a greater CMT change from baseline (SD) at 1 year $(-35[113] \mu \mathrm{m})$ than those who $\operatorname{did} \operatorname{not}(-1[133] \mu \mathrm{m})$.

\section{Discussion}

This retrospective analysis of Taiwanese patients with type 2 diabetes and DME reveals the impact of the introduction (and subsequent reimbursement) of anti-VEGF treatments on DME treatment patterns and health care resource utilization. Furthermore, the results suggest an association between the frequency of anti-VEGF injections and anatomic outcomes. A similar association between the anti-VEGF injection frequency and VA in real-world practice has been hypothesized by investigators, ${ }^{21}$ based on usage data from clinical trials in DME.

Our results highlight a decrease in the use of bevacizumab between 2009 and 2014 that coincided with the increase in ranibizumab use (attributed to its reimbursement in Taiwan 

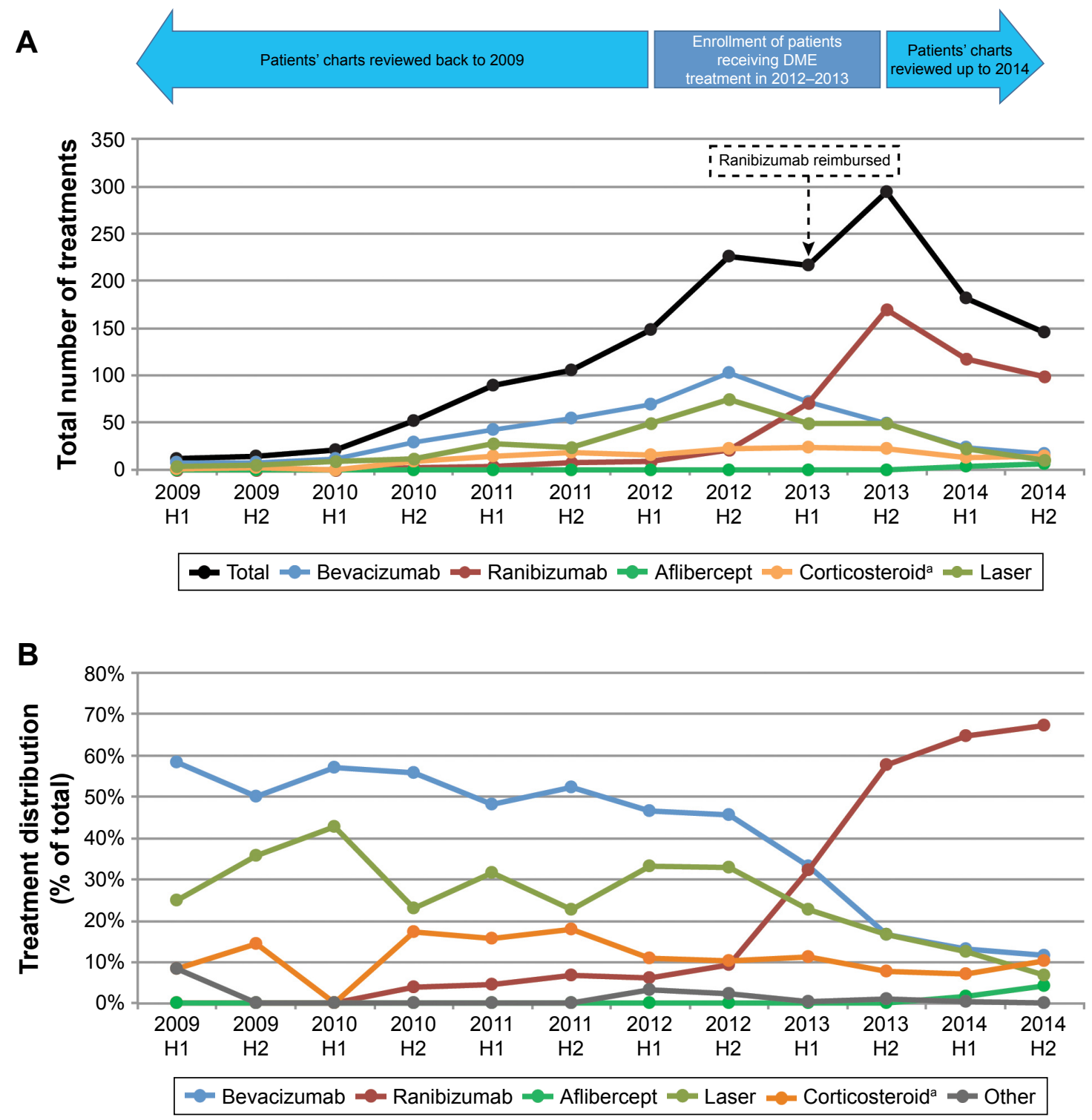

Figure I Number (A) and distribution (B) of DME treatments over time in the prevalent population.

Note: aCorticosteroids included triamcinolone and dexamethasone intravitreal injections.

Abbreviations: DME, diabetic macular edema; $\mathrm{HI}$, months $\mathrm{I}-6$; $\mathrm{H} 2$, months 7-I2.

beginning in February 2013). Furthermore, the use of laser treatment declined, which likely reflects changes in the treatment paradigm of DME with the advent of anti-VEGF agents (as previously reported in a claims-based analysis of the Taiwan National Health Insurance Database from 2004 to 2009). ${ }^{6}$ In contrast, use of off-label intravitreal corticosteroid remained consistently low throughout the study period.

Health care resource utilization data showed that clinic, treatment, and OCT visits were generally more frequent during the first year of treatment, declining in subsequent years. This pattern may reflect both the treatment approach in DME (ie, the treatment schedule is usually more intensive/ frequent early after diagnosis, and less frequent once the disease stabilizes) and patient behavior (ie, poorer compliance over time and/or increase in loss to follow-up leading to fewer visits, as is common with chronic disease ${ }^{22-25}$ ). Health care resource utilization also differed according to the type of therapeutic agent prescribed during the index period; it was highest in patients who received anti-VEGF treatment (alone or in combination with corticosteroid treatment) in the first 6 months post-index, compared with laser or corticosteroids alone, most likely due to the need for more frequent injections and monitoring, a greater disease severity at baseline (data 


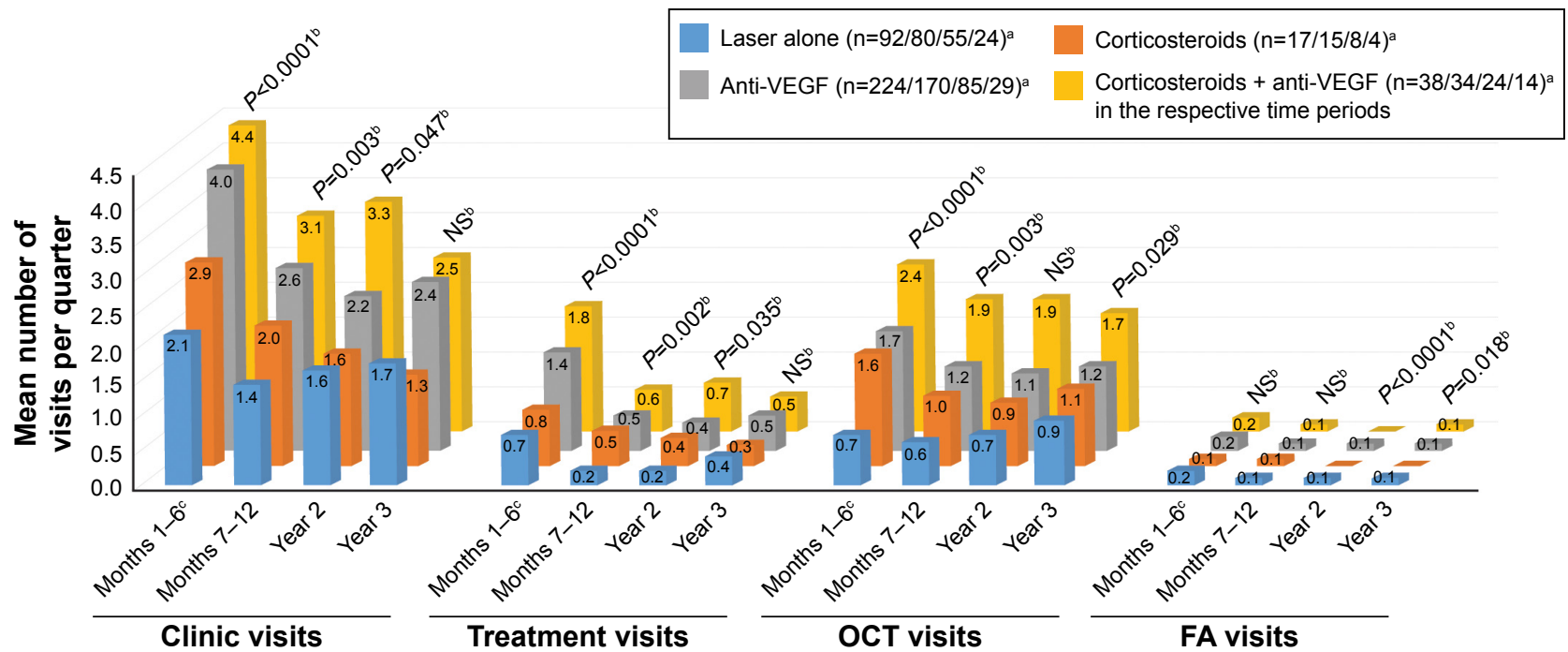

Figure 2 Mean resource utilization per quarter during months 1-6, months 7-12, Year 2, and Year 3 of treatment, categorized by treatment received during the first 6 months after the index date (prevalent population).

Notes: ${ }^{a}$ The four numbers indicate the number of eyes during months I-6, months 7-12, Year 2, and Year 3 periods, respectively. ${ }^{b} P$-values comparing differences across treatment categories were adjusted for age, gender, baseline visual acuity, bilateralism, and number of laser treatments. ${ }^{c} P<0.00 \mathrm{I}$ (months I-6 vs months 7-I2, Year 2, and Year 3). Abbreviations: FA, fluorescein angiography; NS, not significant; OCT, optical coherence tomography; VEGF, vascular endothelial growth factor.

A

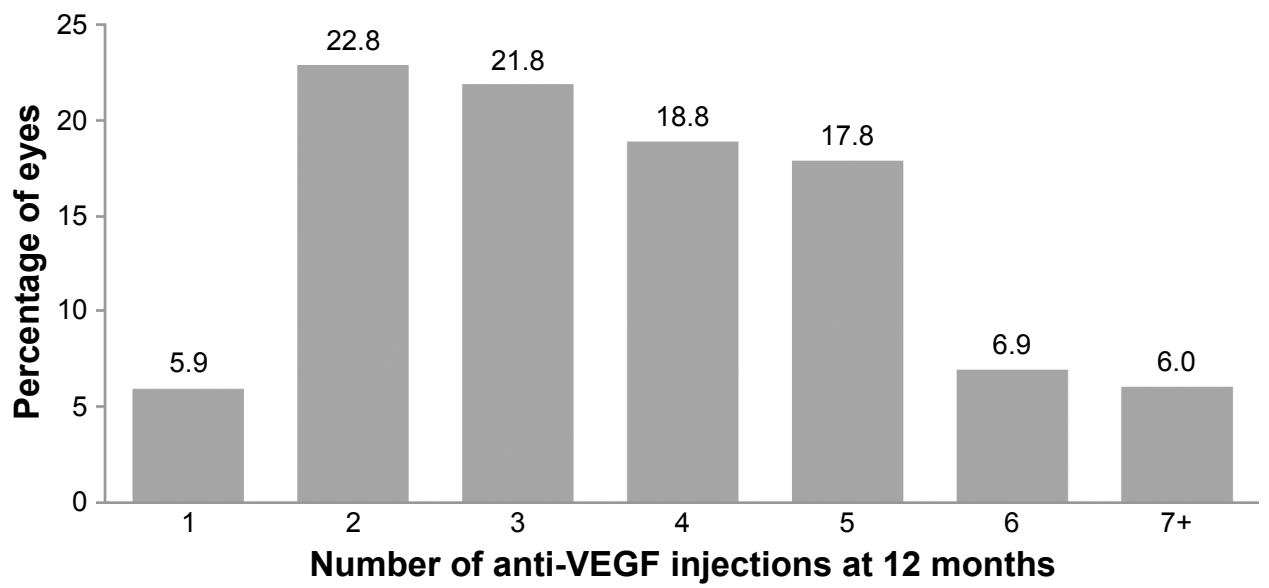

B
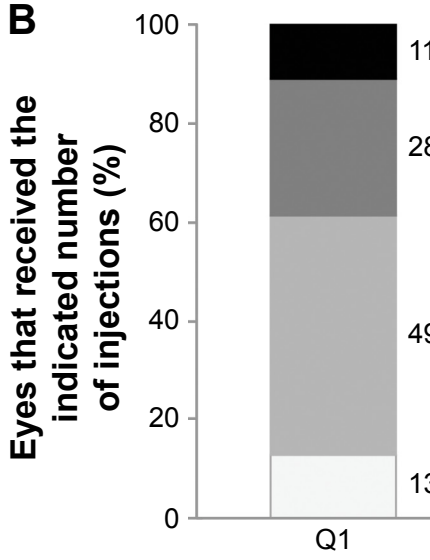

11

28

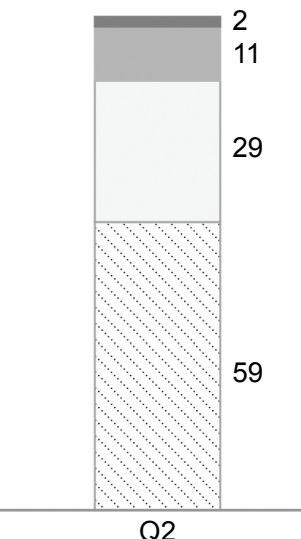

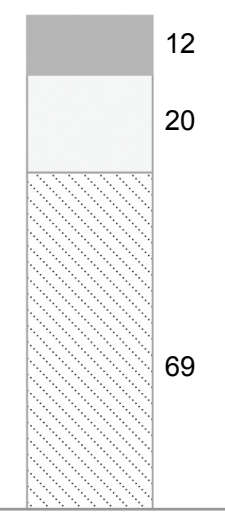

Q3
12

20

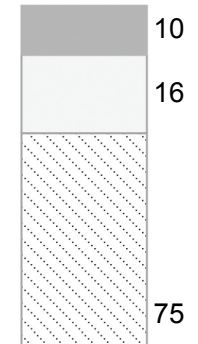

75

\section{Number of anti-VEGF injections at 12 months, per quarter}

$$
\square 0 \square 1 \square 2 \square 3 \square 4
$$

Figure 3 Distribution of the number of anti-VEGF injections administered (A) during the first year post-index, and (B) by quarter during the first year post-index (anti-VEGF-naïve population).

Abbreviations: Q, quarter; VEGF, vascular endothelial growth factor. 


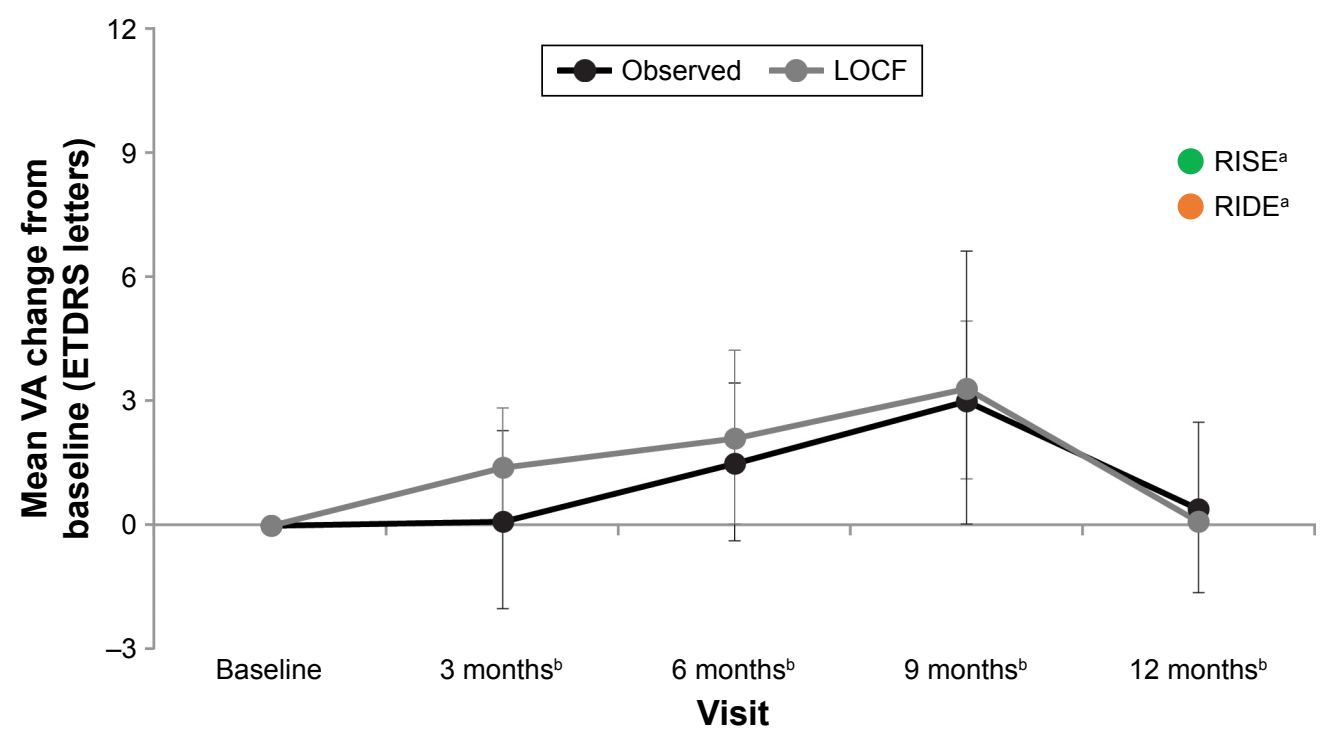

Figure 4 Change in mean (SE) VA from baseline. VA was originally recorded in Snellen and converted to ETDRS letters. ${ }^{18}$ Included in this analysis were patients who had $\geq 12$ months of follow-up data and a VA of $25-70$ letters (similar to the criteria used in published Phase III anti-VEGF trials) ${ }^{12-14}$ between days -30 and +7 and days 60 and 419 of the index date, and did not receive anti-VEGF treatment before 2012 .

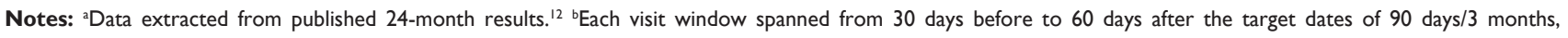
180 days $/ 6$ months, 270 days/9 months, and 365 days/I 2 months. Within each visit window, the VA measurement closest to the target data was used.

Abbreviations: ETDRS, Early Treatment Diabetic Retinopathy Study; LOCF, last observation carried forward; SE, standard error; VA, visual acuity; VEGF, vascular endothelial growth factor.

not shown), and the requirement to demonstrate achievement of specific OCT-based central retinal thickness and VA to continue treatment (per the Taiwanese Bureau of National Health Insurance). In these patients, the mean number of clinic visits during the first year post-index ranged from 6.4 to 6.8 , consistent with a mean of 6.9 clinic visits during the first year recently reported in the LUMINOUS study of ranibizumab in clinical practice. ${ }^{26}$
In the anti-VEGF-naïve population, it is noteworthy that $94 \%$ of study eyes received fewer than seven anti-VEGF injections in the first 12 months of treatment. This finding is in contrast with that of landmark clinical trials of anti-VEGF agents in which patients received seven to ten injections (on average) in the first year of treatment. ${ }^{12,13,15,27,28}$ However, it is consistent with other real-world studies that reported a mean of two to four injections during the first year of

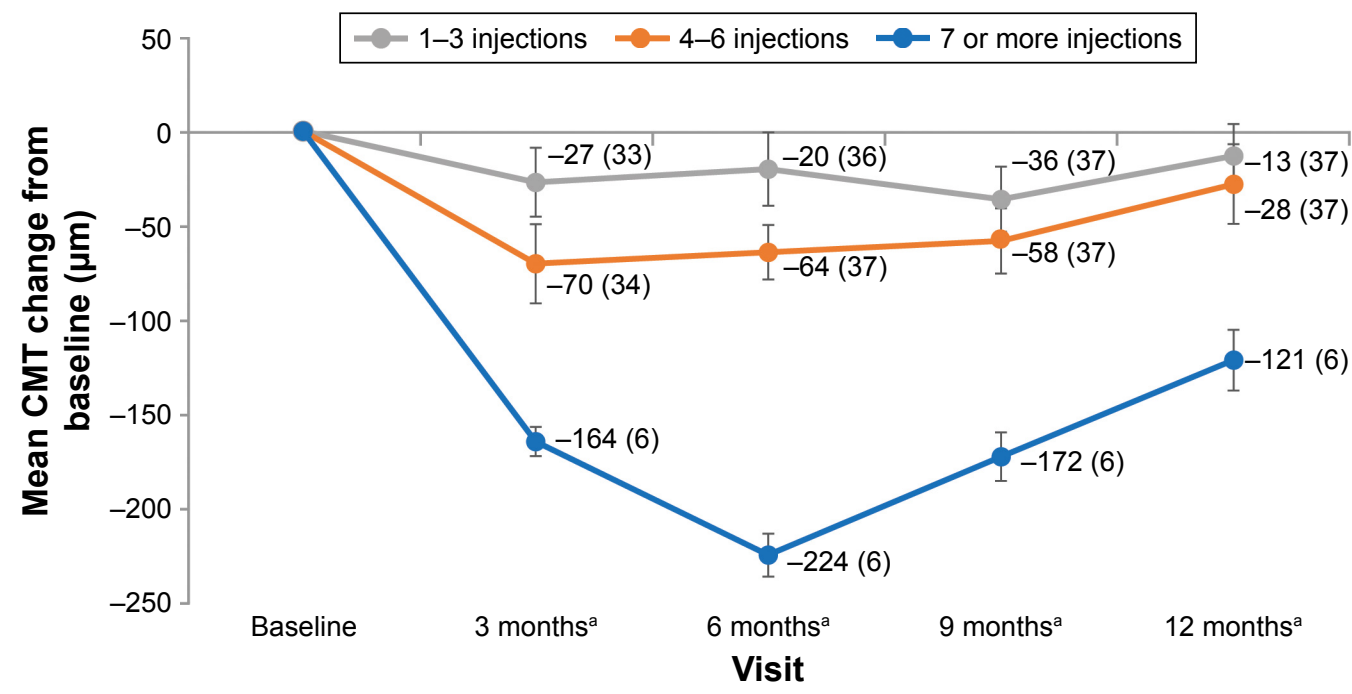

Figure 5 Change in CMT (SE) from baseline (based on the LOCF) categorized by number of anti-VEGF injections in the first year post-index (anti-VEGF-naïve population). The index period spanned from January I, 2012 to December 3I, 20I3. Values shown in parentheses are the number of patients.

Notes: ${ }^{a}$ Each visit window spanned from 30 days before to 60 days after the target dates of 90 days/3 months, 180 days $/ 6$ months, 270 days/9 months, and 365 days/I 2 months. Within each visit window, the CMT measurement closest to the target data was used.

Abbreviations: CMT, central macular thickness; LOCF, last observation carried forward; SE, standard error; VEGF, vascular endothelial growth factor. 
treatment. ${ }^{21,26,29-31}$ This low frequency of anti-VEGF injections is likely due to the already high treatment burden experienced by diabetic patients with DME. ${ }^{32-34}$ A claims database study in the United States showed that patients with DME had significantly more comorbidities, as well as greater health care utilization and treatment burden, compared with diabetic patients with no DME. ${ }^{34}$ The study suggested that the increased burden may force DME patients to choose between obtaining health care and managing their work and other personal commitments. ${ }^{34}$ Indeed, DME patients have to contend with numerous visits to various other medical specialists (eg, diabetes specialist, cardiologist, and nephrologist), ${ }^{33,34}$ which may limit their capacity to accommodate or adhere to frequent ophthalmologist visits. ${ }^{32}$ Furthermore, during the study period, Taiwanese reimbursement guidelines for ranibizumab required demonstration of efficacy after the first three injections (ie, improvement in CMT/VA) in order to receive approval for further treatment, which may have contributed to the limited number of treatments received by patients in this study. In turn, the low number of anti-VEGF injections (mean 3.7) administered in the first year post-index was associated with an observed VA change of 0.4 letters at 12 months ( 0.1 letters after imputation for missing data), compared with gains of 6.1-10.2 letters reported in randomized clinical trials, ${ }^{15,27,28,35}$ suggesting a correlation between the number of injections and visual outcomes. Further research is needed to quantify any such relationship. The lack of association between the number of anti-VEGF treatments and VA gain in this study may be due to the small sample size (especially in the $\geq 7$-injection category), the fact that the number of injections decreased markedly after the first quarter of the year, and/or that VA was not measured at a fixed time-point post-treatment in this real-world clinical study, thus potentially contributing to the measurement variability. The fact that $59 \%$ and $69 \%$ of patients did not receive anti-VEGF injections in the second and third quarter of the first year post-index, respectively, could also explain why the mean changes from baseline in VA and CMT decreased after 9 months, and thus support the need for additional treatments to improve outcomes.

A potential limitation of this study was that health care resource utilization was analyzed based on the type of, and time from, patients' initial treatment, and the impact of change in treatment after 6 months was not explicitly examined. Evaluation of patients who switched treatments would have led to many permutations, as well as sample sizes too small for statistical analysis. Other potential study limitations include the high proportion of patients with center-involved DME, which may have skewed the results toward utilization of antiVEGF agents - the recommended first-line therapy with or without laser. ${ }^{36}$ Because baseline VA was based on measurements obtained from 30 days before to 7 days after the index date, and certain patients experience some visual improvement shortly after an anti-VEGF injection, the minority of patients whose baseline VA was established within that 7-day period may have slightly biased (ie, underestimated) the results. The retrospective nature of the study, missing data (eg, due to loss to follow-up), and the potentially higher data capture from compliant patients (who attend clinic appointments) may have introduced biases as well. In addition, data capture for each patient was limited to one hospital, which does not account for visits and treatments at other hospitals, thereby potentially underestimating the health care resource utilization. However, feedback from treating physicians indicated that the likelihood of "clinic hopping" was low, particularly during the first year of treatment. Finally, there is a possibility that poor outcomes resulted in a decreased injection frequency due to reduced patient motivation and interest in the treatment.

As of February 1, 2016, new reimbursement guidelines in Taiwan allow patients to receive five ranibizumab treatments before demonstration of efficacy is required for approval of further treatments. The impact that this will have on the evolving treatment patterns of DME in Taiwan should be evaluated in future studies.

\section{Conclusion}

Our findings show that, between 2009 and 2014, a change in treatment patterns for DME occurred in Taiwanese hospital ophthalmology clinics after anti-VEGF agents became available. Use of ranibizumab markedly increased following its approval for reimbursement in 2013, whereas use of off-label bevacizumab and traditional laser treatment declined. This study also showed that, in the real-world setting, only a small proportion of patients received anti-VEGF treatment at a frequency similar to that of the landmark Phase III trials. The majority (69\%) of patients received one to four injections over the first 12 months, and this was associated with poorer visual outcomes. Consequently, clinicians should consider beforehand a patient's likelihood and ability to receive the appropriate number of anti-VEGF treatments, as there are alternative, effective DME treatment options available with a lower treatment burden.

\section{Acknowledgments}

We acknowledge the Taiwan Retinal Society for their contribution to this study, including the selection of participating 
clinics, as well as Emily Cheng (Allergan Taiwan Co. Ltd., Taipei, Taiwan) for her contribution to data acquisition, validation, and analysis, and input on the manuscript content. Writing and editorial assistance was provided to the authors by Michele Jacob, PhD, CMPP, of Evidence Scientific Solutions, Inc (Philadelphia, PA, USA), and was funded by Allergan plc (Dublin, Ireland). Neither honoraria nor payments were made for authorship. This work was supported by Allergan Singapore Pte. Ltd. (Singapore). The funding body was involved in the study design, data analysis and interpretation, revision of the manuscript for intellectual content, and decision to submit the manuscript for publication.

\section{Author contributions}

SJS, CKC, HKK, CYT, TCL, and SJC contributed to study conception and design; data acquisition and validation; data analysis and interpretation. JT and HC contributed to data analysis and interpretation. MA contributed to data management and analysis. All authors contributed to drafting and critical revision of the manuscript for important intellectual content, gave final approval of the version to be published, and agreed to be accountable for all aspects of the work.

\section{Disclosure}

CKC, HKK, CYT, and TCL report no competing interests. SJS is a speaker for Allergan, Novartis, and Bayer, and has received research support from Allergan and Novartis. SJC has received grant/research support from Novartis, Bayer, and Allergan; honoraria/consulting fees/reimbursement for travel expenses from Novartis and Bayer; and is a member of an advisory panel, standing committee, and board of directors for Novartis and Bayer. MA is an employee of Datalytics Pty Ltd and a statistical consultant for Allergan, Novartis, and Bayer. HC is an employee of Allergan Singapore Pte. Ltd. JT was an employee of Allergan Singapore Pte. Ltd at the time this study was conducted. The authors report no other conflicts of interest in this work.

\section{References}

1. Shaw JE, Sicree RA, Zimmet PZ. Global estimates of the prevalence of diabetes for 2010 and 2030. Diabetes Res Clin Pract. 2010;87(1): 4-14.

2. Sivaprasad S, Gupta B, Crosby-Nwaobi R, Evans J. Prevalence of diabetic retinopathy in various ethnic groups: a worldwide perspective. Surv Ophthalmol. 2012;57(4):347-370.

3. Nentwich MM, Ulbig MW. Diabetic retinopathy - ocular complications of diabetes mellitus. World J Diabetes. 2015;6(3):489-499.

4. American Academy of Ophthalmology Retina/Vitreous Panel [webpage on the Internet]. Preferred Practice Pattern ${ }^{\circledR}$ Guidelines, Diabetic Retinopathy. San Francisco, CA: American Academy of Ophthalmology; 2016. Available from: https://www.aao.org/preferred-practice-pattern/ diabetic-retinopathy-ppp-updated-2017. Accessed September 1, 2018.
5. World Health Organization [webpage on the Internet]. Prevention of Blindness and Visual Impairment, Priority eye diseases, Diabetic retinopathy. Available from: http://www.who.int/blindness/causes/ priority/en/index 5.html. Accessed September 1, 2018.

6. Lin JC, Shau WY, Lai MS. Sex- and age-specific prevalence and incidence rates of sight-threatening diabetic retinopathy in Taiwan. JAMA Ophthalmol. 2014;132(8):922-928.

7. Romero-Aroca P, de La Riva-Fernandez S, Valls-Mateu A, SagarraAlamo R, Moreno-Ribas A, Soler N. Changes observed in diabetic retinopathy: eight-year follow-up of a Spanish population. Br J Ophthalmol. 2016;100(10):1366-1371.

8. Chen E, Looman M, Laouri M, et al. Burden of illness of diabetic macular edema: literature review. Curr Med Res Opin. 2010;26(7):1587-1597.

9. Yang MC, Tan EC, Su YJ, Lai CC. PSS2 Epidemiology, treatment patterns and medical expenses of diabetic macular edema in Taiwan: a nationwide claim-based database study. Value Health. 2012;15(7): A642.

10. Shea AM, Curtis LH, Hammill BG, et al. Resource use and costs associated with diabetic macular edema in elderly persons. Arch Ophthalmol. 2008;126(12):1748-1754.

11. Lee LJ, Yu AP, Cahill KE, et al. Direct and indirect costs among employees with diabetic retinopathy in the United States. Curr Med Res Opin. 2008;24(5):1549-1559.

12. Nguyen QD, Brown DM, Marcus DM, et al.; for the RISE and RIDE Research Group. Ranibizumab for diabetic macular edema: results from 2 phase III randomized trials: RISE and RIDE. Ophthalmology. 2012; 119(4):789-801.

13. Korobelnik JF, Do DV, Schmidt-Erfurth U, et al. Intravitreal aflibercept for diabetic macular edema. Ophthalmology. 2014;121(11):2247-2254.

14. Ishibashi $\mathrm{T}$, Li X, Koh A, et al. The REVEAL Study: Ranibizumab monotherapy or combined with laser versus laser monotherapy in asian patients with diabetic macular edema. Ophthalmology. 2015; 122(7):1402-1415.

15. Michaelides M, Kaines A, Hamilton RD, et al. A prospective randomized trial of intravitreal bevacizumab or laser therapy in the management of diabetic macular edema (BOLT study) 12-month data: report 2. Ophthalmology. 2010;117(6):1078-1086.

16. Dang Y, Mu Y, Li L, et al. Comparison of dexamethasone intravitreal implant and intravitreal triamcinolone acetonide for the treatment of pseudophakic cystoid macular edema in diabetic patients. Drug Des Devel Ther. 2014;8:1441-1449.

17. Boyer DS, Yoon YH, Belfort R, et al; Ozurdex MEAD Study Group. Three-year, randomized, sham-controlled trial of dexamethasone intravitreal implant in patients with diabetic macular edema. Ophthalmology. 2014;121(10):1904-1914.

18. Gregori NZ, Feuer W, Rosenfeld PJ. Novel method for analyzing snellen visual acuity measurements. Retina. 2010;30(7):1046-1050.

19. Bressler SB, Edwards AR, Andreoli CM, et al; for the Diabetic Retinopathy Clinical Research Network/Writing Committee. Reproducibility of Optovue RTVue optical coherence tomography retinal thickness measurements and conversion to equivalent Zeiss Stratus metrics in diabetic macular edema. Transl Vis Sci Technol. 2015;4(1):5.

20. Bressler SB, Edwards AR, Chalam KV, et al; Diabetic Retinopathy Clinical Research Network Writing Committee. Reproducibility of spectral-domain optical coherence tomography retinal thickness measurements and conversion to equivalent time-domain metrics in diabetic macular edema. JAMA Ophthalmol. 2014;132(9):1113-1122.

21. Kiss S, Liu Y, Brown J, et al. Clinical utilization of anti-vascular endothelial growth-factor agents and patient monitoring in retinal vein occlusion and diabetic macular edema. Clin Ophthalmol. 2014;8: 1611-1621.

22. Brandi ML. New dosing options in osteoporosis treatment: clinical evidence on Risedronate $75 \mathrm{mg}$ monthly treatment. Clin Cases Miner Bone Metab. 2008;5(2):155-158.

23. Cotté FE, Fardellone P, Mercier F, Gaudin AF, Roux C. Adherence to monthly and weekly oral bisphosphonates in women with osteoporosis. Osteoporos Int. 2010;21(1):145-155. 
24. Dunbar-Jacob J, Erlen JA, Schlenk EA, Ryan CM, Sereika SM, Doswell WM. Adherence in chronic disease. Annu Rev Nurs Res. 2000; 18:48-90.

25. Hansen R, Seifeldin R, Noe L. Medication adherence in chronic disease: issues in posttransplant immunosuppression. Transplant Proc. 2007; 39(5):1287-1300.

26. Souied EH, Gekkiev B, Bretagne A, Pilz S, Lacey S. Ranibizumab in a real-world clinical setting: 2014 interim analysis of the 5-year observational multicenter LUMINOUS study. Invest Ophthalmol Vis Sci. 2015;56(7):1417.

27. Elman MJ, Aiello LP, Beck RW, et al; Diabetic Retinopathy Clinical Research Network. Randomized trial evaluating ranibizumab plus prompt or deferred laser or triamcinolone plus prompt laser for diabetic macular edema. Ophthalmology. 2010;117(6):1064-1077.

28. Massin P, Bandello F, Garweg JG, et al. Safety and efficacy of ranibizumab in diabetic macular edema (RESOLVE Study): a 12-month, randomized, controlled, double-masked, multicenter phase II study. Diabetes Care. 2010;33(11):2399-2405.

29. Campbell J, Cole A, Almony A, et al. Real world vision outcomes in DME treated with anti-VEGF injections - an analysis of EMR data from a large health system. Invest Ophthalmol Vis Sci. 2014; 55(13):3065.

30. Holekamp N, Dugel P, Yep T, et al. Utilization of anti-VEGFs and dexamethasone intravitreal implant for diabetic macular edema in US clinical practice using the Vestrum Health ${ }^{\mathrm{TM}}$ Database. Presentation at: Retina Society 48th Annual Meeting; October 7-11; 2015; Paris, France.
31. Jiang S, Barner JC, Park C, Ling YL. Treatment patterns of antivascular endothelial growth factor and laser therapy among patients with diabetic macular edema. J Manag Care Spec Pharm. 2015;21(9): 735-741.

32. Virnig BA, Shippee ND, O’Donnell B, Zeglin J, Parashuram S. Use of and access to health care by Medicare beneficiaries with diabetes: impact of diabetes type and insulin use, 2007-2011. Use of Diabetes Care. Data Points \# 18. Data Points Publication Series. Rockville, MD: Agency for Healthcare Research and Quality; 2014.

33. Wallick C, Hansen R, Campbell J, Kowalski J, Kiss S, Sullivan S. Increased health care utilization among patients with diabetic macular edema compared with diabetic patients without edema [abstract]. Invest Ophthalmol Vis Sci. 2014;55:1751.

34. Wallick CJ, Hansen RN, Campbell J, Kiss S, Kowalski JW, Sullivan SD. Comorbidity and health care resource use among commercially insured non-elderly patients with diabetic macular edema. Ophthalmic Surg Lasers Imaging Retina. 2015;46(7):744-751.

35. Mitchell P, Bandello F, Schmidt-Erfurth U, et al; RESTORE study group. The RESTORE study: ranibizumab monotherapy or combined with laser versus laser monotherapy for diabetic macular edema. Ophthalmology. 2011;118(4):615-625.

36. Ghanchi F; Diabetic Retinopathy Guidelines Working Group. The Royal College of Ophthalmologists' clinical guidelines for diabetic retinopathy: a summary. Eye. 2013;27(2):285-287.
Clinical Ophthalmology

\section{Publish your work in this journal}

Clinical Ophthalmology is an international, peer-reviewed journal covering all subspecialties within ophthalmology. Key topics include: Optometry; Visual science; Pharmacology and drug therapy in eye diseases; Basic Sciences; Primary and Secondary eye care; Patient Safety and Quality of Care Improvements. This journal is indexed on

\section{Dovepress}

PubMed Central and CAS, and is the official journal of The Society of Clinical Ophthalmology (SCO). The manuscript management system is completely online and includes a very quick and fair peer-review system, which is all easy to use. Visit http://www.dovepress.com/ testimonials.php to read real quotes from published authors. 